\title{
Determinants of mortality in elderly patients with tuberculosis: a population-based follow-up study
}

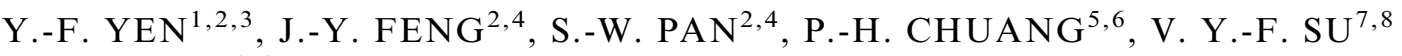 \\ AND W.-J. SU ${ }^{2,4 *}$ \\ ${ }^{1}$ Section of Infectious Diseases, Taipei City Hospital, Taipei City Government, Taipei, Taiwan \\ ${ }^{2}$ School of Medicine, National Yang-Ming University, Taipei, Taiwan \\ ${ }^{3}$ Department of Health and Welfare, College of City Management, University of Taipei, Taiwan \\ ${ }^{4}$ Department of Chest Medicine, Taipei Veterans General Hospital, Taipei, Taiwan \\ ${ }^{5}$ Center for Prevention and Treatment of Occupational Injury and Diseases, Taipei veterans General Hospital, \\ Taipei, Taiwan \\ ${ }^{6}$ Department of Medicine, Division of Clinical Toxicology and Occupational Medicine, Taipei Veterans General \\ Hospital, Taipei, Taiwan \\ ${ }^{7}$ Department of Critical Care Medicine, Taipei Veterans General Hospital, Taipei, Taiwan \\ ${ }^{8}$ Institute of Clinical Medicine, National Yang-Ming University, Taipei, Taiwan
}

Received 16 October 2016; Final revision 19 December 2016; Accepted 7 January 2017; first published online 13 February 2017

\section{SUMMARY}

Elderly individuals with tuberculosis (TB) are more likely to have a non-specific clinical presentation of TB and high mortality. However, factors associated with mortality in elderly TB patients have not been extensively studied. This retrospective cohort study aimed to identify factors associated with death among elderly Taiwanese with TB. All elderly patients with TB from 2006 to 2014 in Taipei, Taiwan, were included in a study. Multiple logistic regression was used to identify the factors associated with death in elderly TB patients. The mean age of the 5011 patients was $79 \cdot 7$ years; $74 \cdot 1 \%$ were men; $32.7 \%$ had mortality during the study follow-up period. After controlling for potential confounders, age $\geqslant 75$ years (reference: $65-74$ years), male sex, end-stage renal disease (ESRD), malignancy, acid-fast bacilli-smear positivity, TB-culture positivity, pleural effusion on chest radiograph and notification by an ordinary ward or intensive care unit were associated with a higher risk of all-cause death; while high school, and university or higher education, cavity on chest radiograph and directly observed therapy were associated with a lower risk of all-cause death. This study found that the proportion of death among elderly patients with TB in Taipei, Taiwan, was high. To improve TB treatment outcomes, future control programmes should particularly target individuals with comorbidities (e.g. ESRD and malignancy) and those with a lower socio-economic status (e.g. not educated).

Key words: Elderly, mortality, Taiwan, tuberculosis.

\section{INTRODUCTION}

Approximately one-third of the world's population is latently infected with tuberculosis (TB). In 2014, there were an estimated $9 \cdot 6$ million incident cases of $\mathrm{TB}$, and 1.5 million people died from the disease [1].

\footnotetext{
* Author for correspondence: W.-J. Su, MD, MPH, Department of Chest Medicine, Taipei Veterans General Hospital, Taipei, Taiwan, Republic of China

(Email: wjsu@vghtpe.gov.tw)
} 
In Taiwan, of all notifiable infectious diseases, TB has been the most prevalent for decades [2]. Since 2006, Taiwan's Centers for Disease Control and Prevention (CDC) has adopted a directly observed therapy short-course (DOTS) programme to halve TB incidence and achieve a successful treatment rate of $85 \%$ by 2015 . Since then, the rate of TB incidence has decreased from $72 \cdot 5$ per 100000 in 2005 to $48 \cdot 4$ in 2014 [2]. The success rate for TB treatment, however, only slightly improved from $64 \cdot 1 \%$ in 2005 to $70.4 \%$ in 2014 . Mortality among Taiwanese patients with TB accounted for $81.8 \%$ of the cases of unsuccessful TB treatment [2]. Additionally, over $82 \cdot 1 \%$ of TB deaths in Taiwan were among individuals $>65$ years old [3].

TB in the elderly ( $>65$ years old) has increasingly become a major issue not only because of non-specific clinical presentation of TB but, more importantly, because of a high mortality rate $[4,5]$. Since the clinical symptoms of TB and risk factors of mortality in older patients may differ from those in younger patients, TB diseases in this population should be classified as a separate entity [6, 7]. Few studies, however, have determined the factors associated with TB mortality among elderly patients [7].

According to the World Health Organization, death is defined as a patient who dies for any reason during treatment [8]. However, many patients with TB do not die of TB but from other causes such as malignancy or end-stage renal disease (ESRD). A previous review article found that few studies about TB treatment outcomes distinguished TB-specific mortality from other-cause (non-TB-specific) mortality [8]. Developing effective interventions to improve TB outcomes requires better understanding of the factors associated with mortality, particularly for vulnerable populations. We thus attempted to identify prognostic factors associated with mortality among elderly Taiwanese patients with TB infection from 2006 to 2014.

\section{METHODS}

\section{Study population and data source}

This retrospective cohort study used TB surveillance data collected by the Taipei City Government in Taiwan. The subjects included were elderly Taiwanese individuals (age $\geqslant 65$ years) with $\mathrm{TB}$ in Taipei from 2006 to 2014. In Taiwan, all suspected TB cases must be reported to Taiwan's CDC within
7 days [2]. After receiving notification, trained case managers use a structured questionnaire to interview patients about their socio-demographic characteristics, clinical findings, underlying diseases, admission history and TB treatments. Patients with TB in Taipei are required by law to be monitored until treatment success, death, or loss to follow-up. For the purpose of monitoring treatment response, case managers followed up all TB cases by phone or in person once every other week. This project was approved by the Institutional Review Board of Taipei City Hospitals (TCHIRB-10505112-E).

\section{Outcome variables}

The outcome variable of interest was treatment outcome, which was categorised into two groups: successful treatment and mortality. Mortality was classified as TB-specific or non-TB-specific death, which was determined by the Taiwan Death Certification Registry [9]. TB-specific death in this study was defined as the underlying cause of death being due to TB according to the Taiwan Death Certification Registry (International Classification of Diseases, 9th Revision, Clinical Modification (ICD-9-CM) codes: A010-A018; ICD-10-CM code: A15-A19). Non-TB-specific death was defined as any underlying cause of death other than TB.

\section{Explanatory variables}

Covariates identified in previous studies as risk factors for TB mortality or as major comorbidities linked to TB mortality were assessed in the analyses [10], including demographic factors (age, sex, marital status, education level, nursing home residence, smoking, alcohol use and employment status); clinical findings (chest radiography findings, acid-fast bacilli (AFB) smear status, drug resistance); underlying diseases (malignancy and ESRD); source of notification and mode of treatment. The source of the notification was defined as the department that reported the TB case, including ordinary wards, intensive care units or outpatient services. Treatment mode was categorised as directly observed therapy or self-administered therapy. Directly observed therapy was defined as anti-TB medication ingestion that was directly supervised by a trained observer [11]. Self-administered therapy referred to unsupervised treatment. 


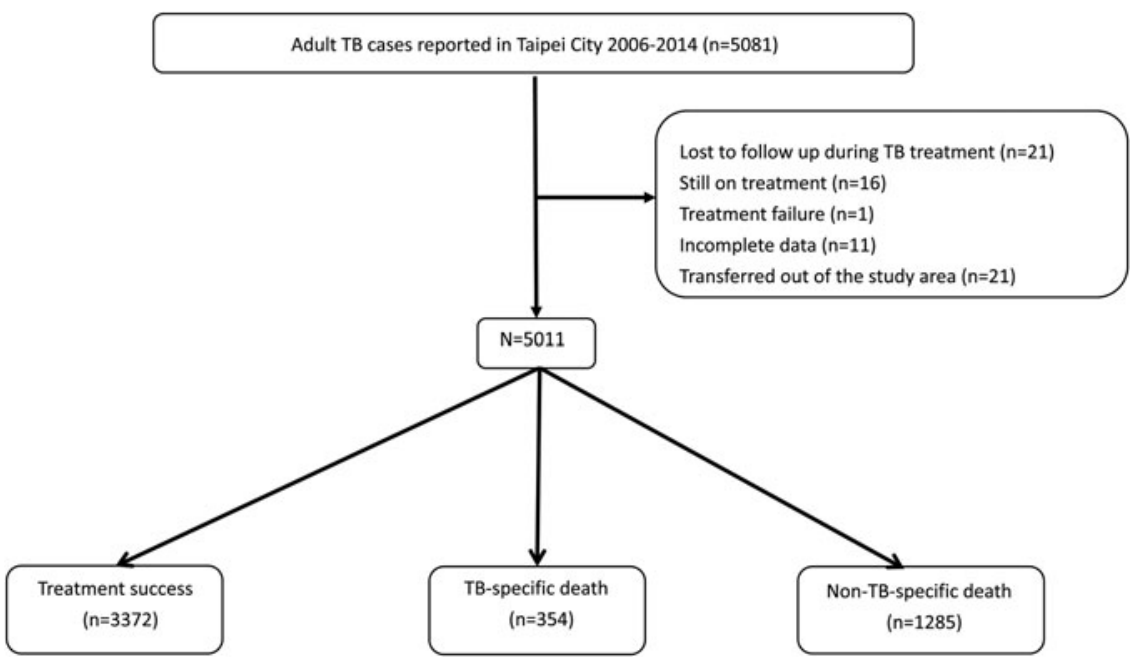

Fig. 1. Study flow diagram. TB, tuberculosis.

\section{Statistical analysis}

In univariate analysis, the $\chi^{2}$ test was used to assess associations of the clinical relevant variables with the outcomes. All variables found to be significant $(P<$ $0.05)$ through univariate analysis were considered for inclusion in multivariate analysis. Multiple logistic regression was used to assess the multivariate associations of the factors with all-cause mortality. Furthermore, multinomial logistic regression was used to identify the factors associated with TB-specific and non-TB-specific mortality. Adjusted odds ratios (AOR) with 95\% confidence intervals $(\mathrm{CI})$ were reported to show the strength and direction of these associations. All data management and analyses were performed using the SAS 9.4 software package (SAS Institute, Cary, NC).

\section{RESULTS}

\section{Characteristics of patients with TB}

During the 9-year study period, there were $5081 \mathrm{~TB}$ cases (age $\geqslant 65$ years) reported to the Taipei TB Control Department. Of these, 21 were lost to follow up, 16 were still on treatment, 1 had treatment failure, 11 had incomplete data and 21 had transferred out of Taipei City (Fig. 1). The remaining 5011 were included in the subsequent analysis. Overall, the mean and median ages of the study subjects were 79.7 years (range 65-113) and 80 years (interquartile range $74-85$ ), respectively; $74 \cdot 1 \%$ were men; $67 \cdot 3 \%$ (3372) were successfully treated; $7 \cdot 1 \%$ (354) died of TB-specific causes and $25.6 \%$ (1285) died of non-TB-specific causes during TB treatment.
The TB-specific mortality rates were $3 \cdot 3 \%, 5 \cdot 7 \%$ and $12 \cdot 7 \%$ in patients aged $65-74,75-84$ and $\geqslant 85$ years, respectively. Additionally, the non-TB-specific mortality rates were $14 \cdot 0 \%, 26 \cdot 0 \%$ and $36 \cdot 2 \%$ in patients aged $65-74, \quad 75-84$ and $\geqslant 85$ years, respectively.

\section{Univariate analyses of factors associated with all-cause mortality}

The $\chi^{2}$ test revealed variables that were significantly associated with all-cause mortality, i.e. age $75-84$ or $\geqslant 85$ years, unemployment, residence in a long-term care facility, ESRD, malignancy, AFB-smear positivity, TB-culture positivity, pleural effusion on chest radiograph and notification from an ordinary ward or intensive care unit (Table 1). Additionally, variables significantly associated with a lower chance of all-cause mortality were elementary school, high school, and university or higher education, a cavity on chest radiograph and directly observed therapy.

\section{Multivariates analyses of factors associated with all-cause mortality}

All variables found to be significant $(P<0.05)$ through univariate analysis were considered for inclusion in multivariate analysis. After adjusting for sociodemographic factors and comorbidities, risk factors associated with all-cause mortality included age 75-84 years $(\mathrm{AOR}=2 \cdot 18,95 \%$ CI $1 \cdot 82-2 \cdot 61)$, age $\geqslant 85$ years (AOR $=4 \cdot 57,95 \%$ CI $3 \cdot 78-5 \cdot 53)$, residence in a longterm care facility $(\mathrm{AOR}=1 \cdot 57,95 \%$ CI $1 \cdot 22-2 \cdot 01)$, 
Table 1. Univariate analyses of risk factors for mortality in elderly TB patients, Taipei, Taiwan (2006-2014)

\begin{tabular}{|c|c|c|c|}
\hline \multirow[b]{2}{*}{ Factor } & \multirow[b]{2}{*}{ Number of patients } & \multirow{2}{*}{$\frac{\text { All-cause deaths }}{n(\%)}$} & \multirow{2}{*}{$\frac{\text { Univariate analysi }}{\text { OR }(95 \% \mathrm{CI})}$} \\
\hline & & & \\
\hline \multicolumn{4}{|l|}{ Age (years) } \\
\hline $65-74$ & 1389 & $241(17 \cdot 4)$ & 1 \\
\hline $75-84$ & 2168 & $686(31 \cdot 6)$ & $2 \cdot 21(1 \cdot 87-2 \cdot 60)^{* * *}$ \\
\hline$\geqslant 85$ & 1454 & $712(49 \cdot 0)$ & $4 \cdot 57(3 \cdot 85-5 \cdot 43)^{* * *}$ \\
\hline \multicolumn{4}{|l|}{ Sex } \\
\hline Female & 1299 & $401(30 \cdot 9)$ & 1 \\
\hline Male & 3712 & $1238(33 \cdot 4)$ & $1 \cdot 12(0 \cdot 98-1 \cdot 29)$ \\
\hline \multicolumn{4}{|l|}{ Marital status } \\
\hline Unmarried & 378 & $134(35 \cdot 5)$ & 1 \\
\hline Married & 4633 & $1505(32 \cdot 5)$ & $0 \cdot 88(0 \cdot 70-1 \cdot 09)$ \\
\hline \multicolumn{4}{|l|}{ Education level } \\
\hline No education & 656 & $253(38 \cdot 6)$ & 1 \\
\hline Elementary school & 1425 & $473(33 \cdot 2)$ & $0.79(0 \cdot 65-0 \cdot 96)^{*}$ \\
\hline High school & 1485 & $434(29 \cdot 2)$ & $0.66(0.54-0.80)^{* * *}$ \\
\hline University or higher & 921 & $260(28 \cdot 2)$ & $0.63(0.51-0.78)^{* * *}$ \\
\hline Unknown & 524 & $219(41 \cdot 8)$ & $1 \cdot 14(0 \cdot 91-1 \cdot 45)$ \\
\hline \multicolumn{4}{|l|}{ Smoker } \\
\hline No & 4497 & $1485(33 \cdot 0)$ & 1 \\
\hline Yes & 514 & $154(30 \cdot 0)$ & $0 \cdot 87(0 \cdot 71-1 \cdot 06)$ \\
\hline \multicolumn{4}{|l|}{ Unemployment } \\
\hline No & 537 & $144(26 \cdot 8)$ & 1 \\
\hline Yes & 4474 & $1495(33 \cdot 3)$ & $1.37(1.12-1 \cdot 67)^{* *}$ \\
\hline \multicolumn{4}{|l|}{ LTCF residences } \\
\hline No & 4687 & $1474(31 \cdot 5)$ & 1 \\
\hline Yes & 324 & $165(50 \cdot 9)$ & $2 \cdot 26(1 \cdot 80-2 \cdot 84)^{* * *}$ \\
\hline \multicolumn{4}{|l|}{ DM } \\
\hline No & 3995 & $1331(33 \cdot 3)$ & 1 \\
\hline Yes & 1016 & $308(30 \cdot 3)$ & $0 \cdot 87(0 \cdot 75-1 \cdot 01)$ \\
\hline \multicolumn{4}{|l|}{ ESRD } \\
\hline No & 4806 & $1535(31 \cdot 9)$ & 1 \\
\hline Yes & 205 & $104(50 \cdot 7)$ & $2 \cdot 19(1 \cdot 66-2 \cdot 90)^{* * *}$ \\
\hline \multicolumn{4}{|l|}{ Malignancy } \\
\hline No & 4538 & $1392(30 \cdot 7)$ & 1 \\
\hline Yes & 473 & $247(52 \cdot 2)$ & $2.47(2 \cdot 04-2.99)^{* * *}$ \\
\hline \multicolumn{4}{|l|}{ TB relapse } \\
\hline No & 4846 & $1591(32 \cdot 8)$ & 1 \\
\hline Yes & 165 & $48(29 \cdot 1)$ & $0 \cdot 84(0 \cdot 60-1 \cdot 18)$ \\
\hline \multicolumn{4}{|l|}{ Acid-fast bacilli smear } \\
\hline Negative & 3099 & $955(30 \cdot 8)$ & 1 \\
\hline Positive & 1912 & $684(35 \cdot 8)$ & $1.25(1 \cdot 11-1 \cdot 41)^{* * *}$ \\
\hline \multicolumn{4}{|l|}{ TB culture } \\
\hline Negative & 1261 & $359(28 \cdot 5)$ & 1 \\
\hline Positive & 3750 & $1280(34 \cdot 1)$ & $1.30(1.13-1 \cdot 50)^{* * *}$ \\
\hline \multicolumn{4}{|l|}{ Cavities on CXR } \\
\hline No & 4434 & $1479(33 \cdot 4)$ & 1 \\
\hline Yes & 577 & $160(27 \cdot 7)$ & $0.77(0.63-0.93)^{* *}$ \\
\hline \multicolumn{4}{|l|}{ Pleural effusion on CXR } \\
\hline No & 4282 & $1348(31 \cdot 5)$ & 1 \\
\hline Yes & 729 & $291(39 \cdot 9)$ & $1.45(1.23-1 \cdot 70)^{* * *}$ \\
\hline Extrapulmonary TB & & & \\
\hline No & 4638 & $1523(32 \cdot 8)$ & 1 \\
\hline Yes & 373 & $116(31 \cdot 1)$ & $0 \cdot 92(0 \cdot 74-1 \cdot 16)$ \\
\hline MDR-TB & & & \\
\hline No & 4990 & $1634(32 \cdot 8)$ & 1 \\
\hline
\end{tabular}


Table 1 (cont.)

\begin{tabular}{|c|c|c|c|}
\hline \multirow[b]{2}{*}{ Factor } & \multirow[b]{2}{*}{ Number of patients } & \multirow{2}{*}{$\frac{\text { All-cause deaths }}{n(\%)}$} & \multirow{2}{*}{$\frac{\text { Univariate analysis }}{\text { OR }(95 \% \mathrm{CI})}$} \\
\hline & & & \\
\hline Yes & 21 & $5(23 \cdot 8)$ & $0 \cdot 64(0 \cdot 23-1 \cdot 76)$ \\
\hline \multicolumn{4}{|l|}{ Mode of treatment } \\
\hline SAT & 1573 & $659(41 \cdot 9)$ & 1 \\
\hline DOT & 3438 & $980(28 \cdot 5)$ & $0.55(0.49-0.63)^{* * *}$ \\
\hline \multicolumn{4}{|l|}{ Source of notification } \\
\hline Outpatient services & 2413 & $513(21 \cdot 3)$ & 1 \\
\hline Ordinary ward & 2410 & $994(41 \cdot 2)$ & $2 \cdot 60(2 \cdot 29-2 \cdot 95)^{* * *}$ \\
\hline Intensive care unit & 188 & $132(70 \cdot 2)$ & $8.73(6 \cdot 29-2 \cdot 95)^{* * *}$ \\
\hline
\end{tabular}

OR, odds ratio; CI, confidence interval; LTCF, long-term care facility; DM, diabetes mellitus; ESRD, end-stage renal disease; TB, tuberculosis; CXR, chest radiograph; MDR-TB, multidrug-resistant tuberculosis; SAT, self-administrated treatment; DOT, directly observed treatment.

$*<0 \cdot 05 ; * *<0 \cdot 01 ; * * *<0 \cdot 001$.

Table 2. Multivariates analyses of risk factors for all-cause mortality in elderly TB patients, Taipei, Taiwan (2006-2014)

\begin{tabular}{ll}
\hline \hline Factors & AOR $(95 \% \mathrm{CI})$ \\
\hline Age (years) & \\
$\quad 65-74$ & 1 \\
$75-84$ & $2 \cdot 18(1 \cdot 82-2 \cdot 61)^{* * *}$ \\
$\quad$ 285 & $4 \cdot 57(3 \cdot 78-5 \cdot 53)^{* * *}$ \\
Education level & 1 \\
$\quad$ No education & $0 \cdot 91(0 \cdot 74-1 \cdot 13)$ \\
Elementary school & $0 \cdot 72(0 \cdot 58-0 \cdot 89)^{* *}$ \\
High school & $0 \cdot 64(0 \cdot 51-0 \cdot 81)^{* * *}$ \\
University or higher & $1 \cdot 02(0 \cdot 79-1 \cdot 32)$ \\
$\quad$ Unknown & $1 \cdot 57(1 \cdot 22-2 \cdot 01)^{* * *}$ \\
LTCF residences & $0 \cdot 95(0 \cdot 76-1 \cdot 19)$ \\
Unemployment & $2 \cdot 63(1 \cdot 92-3 \cdot 60)^{* * *}$ \\
ESRD & $3 \cdot 01(2 \cdot 43-3 \cdot 74)^{* * *}$ \\
Malignancy & $1 \cdot 59(1 \cdot 37-1 \cdot 85)^{* * *}$ \\
AFB-smear positivity & $1 \cdot 53(1 \cdot 29-1 \cdot 81)^{* * *}$ \\
TB-culture positivity & $0 \cdot 76(0 \cdot 61-0 \cdot 95)^{*}$ \\
Cavity on CXR & $1 \cdot 40(1 \cdot 16-1 \cdot 69)^{* * *}$ \\
Pleural effusion & $0 \cdot 34(0 \cdot 29-0 \cdot 39)^{* * *}$ \\
DOT & 1 \\
Source of notification & $2 \cdot 50(2 \cdot 17-2 \cdot 88)^{* * *}$ \\
Outpatient services & $6 \cdot 71(4 \cdot 72-9 \cdot 54)^{* * *}$ \\
Ordinary ward & \\
Intensive care unit & \\
\hline \hline
\end{tabular}

TB, tuberculosis; AOR, adjusted odds ratio; CI, confidence interval; LTCF, long-term care facility; ESRD, end-stage renal disease; AFB, acid-fast bacilli; CXR, chest radiograph; DOT, directly observed treatment.

$*<0 \cdot 05 ; * *<0 \cdot 01 ; * * *<0 \cdot 001$.
ESRD $(\mathrm{AOR}=2 \cdot 63,95 \%$ CI $1 \cdot 92-3 \cdot 60)$, malignancy (AOR $=3 \cdot 01,95 \%$ CI 2.43-3.74), AFB-smear positivity $(\mathrm{AOR}=1 \cdot 59,95 \% \mathrm{CI} 1 \cdot 37-1 \cdot 85)$, TB-culture positivity $(\mathrm{AOR}=1.53, \quad 95 \%$ CI $1.29-1 \cdot 81)$, pleural effusion on chest radiograph $(\mathrm{AOR}=1 \cdot 40,95 \% \mathrm{CI}$ $1 \cdot 16-1 \cdot 69)$ and notification from an ordinary ward (AOR $=2 \cdot 50,95 \%$ CI $2 \cdot 17-2 \cdot 88$ ) or intensive care unit $(\mathrm{AOR}=6 \cdot 71,95 \%$ CI 4.72-9.54) (Table 2). Additionally, protective factors for all-cause mortality included a high school education $(\mathrm{AOR}=0.72,95 \%$ CI 0.58-0.89), a university or higher education (AOR $=0 \cdot 64,95 \%$ CI $0 \cdot 51-0 \cdot 81)$, a cavity on chest radiograph (AOR $=0 \cdot 76,95 \%$ CI $0 \cdot 61-0 \cdot 95)$ and directly observed therapy $(\mathrm{AOR}=0 \cdot 34,95 \%$ CI 0.29-0.39).

\section{Factors associated with TB-specific and non-TB-specific mortality}

Multinomial regression showed that, after controlling for other variables, risk factors associated with TB-specific and non-TB-specific mortality included age $\geqslant 75$ years, residence in a long-term care facility, ESRD, AFB-smear positivity, TB-culture positivity and notification from an ordinary ward or intensive care unit (Table 3). Protective factors for TB-specific and non-TB-specific mortality included directly observed therapy. Additionally, malignancy and pleural effusion on chest radiographs were significantly associated with a higher risk of non-TB-specific mortality; while high school, university or higher education and a cavity on chest radiograph were significantly associated with a lower risk of non-TB-specific mortality. 
Table 3. Multinomial regression: demographic and clinical variables associated with TB-specific and non-TB-specific death among elderly TB patients in Taipei, Taiwan (2006-2014) ${ }^{a}$

\begin{tabular}{|c|c|c|}
\hline Factors & $\begin{array}{l}\text { TB-specific death } \\
\text { AOR }(95 \% \mathrm{CI})\end{array}$ & $\begin{array}{l}\text { Non-TB-specific death } \\
\text { AOR }(95 \% \mathrm{CI})\end{array}$ \\
\hline \multicolumn{3}{|l|}{ Age (years) } \\
\hline $65-74$ & 1 & 1 \\
\hline $75-84$ & $2 \cdot 01(1 \cdot 41-2 \cdot 87)^{* * *}$ & $2.22(1.83-2.69)^{* * *}$ \\
\hline$\geqslant 85$ & $5.94(4 \cdot 18-8 \cdot 44)^{* * *}$ & $4 \cdot 22(3 \cdot 43-5 \cdot 18)^{* * *}$ \\
\hline \multicolumn{3}{|l|}{ Education level } \\
\hline No education & 1 & 1 \\
\hline Elementary school & $0 \cdot 89(0 \cdot 62-1 \cdot 28)$ & $0 \cdot 92(0 \cdot 73-1 \cdot 16)$ \\
\hline High school & $0 \cdot 70(0 \cdot 49-1 \cdot 01)$ & $0.72(0.57-0.91)^{* *}$ \\
\hline University or higher & $0 \cdot 76(0 \cdot 51-1 \cdot 13)$ & $0.61(0.47-0.79)^{* * *}$ \\
\hline Unknown & $0 \cdot 73(0 \cdot 46-1 \cdot 16)$ & $1 \cdot 11(0 \cdot 84-1 \cdot 46)$ \\
\hline LTCF residences & $1.84(1.25-2.70)^{* *}$ & $1.50(1.15-1.96)^{* *}$ \\
\hline Unemployment & $0.93(0.62-1.39)$ & $0.95(0.75-1.22)$ \\
\hline ESRD & $2.34(1.37-4.00)^{* *}$ & $2.70(1.95-3.74)^{* * *}$ \\
\hline Malignancy & $1 \cdot 21(0 \cdot 77-1 \cdot 90)$ & $3 \cdot 53(2 \cdot 83-4 \cdot 40)^{* * *}$ \\
\hline AFB-smear positivity & $2 \cdot 49(1.91-3 \cdot 23)^{* * *}$ & $1.40(1.19-1.65)^{* * *}$ \\
\hline TB-culture positivity & $1 \cdot 74(1.27-2 \cdot 38)^{* * *}$ & $1.49(1.24-1.78)^{* * *}$ \\
\hline Cavity on CXR & $1.07(0.76-1.51)$ & $0.68(0.53-0.86)^{* *}$ \\
\hline Pleural effusion & $1 \cdot 30(0 \cdot 92-1 \cdot 83)$ & $1.43(1.17-1.74)^{* * *}$ \\
\hline DOT & $0.31(0.24-0.41)^{* * *}$ & $0.34(0 \cdot 29-0.40)^{* * *}$ \\
\hline \multicolumn{3}{|l|}{ Source of notification } \\
\hline Outpatient services & 1 & 1 \\
\hline Ordinary ward & $2 \cdot 96(2 \cdot 27-3 \cdot 87)^{* * *}$ & $2.39(2.06-2.79)^{* * *}$ \\
\hline Intensive care unit & $8 \cdot 66(5 \cdot 23-14.33)^{* * *}$ & $6 \cdot 25(4.32-9.04)^{* * *}$ \\
\hline
\end{tabular}

TB, tuberculosis; AOR, adjusted odds ratio; CI, confidence interval; LTCF, long-term care facility; ESRD, end-stage renal disease; AFB, acid-fast bacilli; CXR, chest radiograph; DOT, directly observed treatment.

${ }^{\text {a }}$ Reference is successfully treated individuals.

$* *<0 \cdot 01 ; * * *<0 \cdot 001$.

\section{DISCUSSION}

In this large cohort study of $5011 \mathrm{~TB}$ cases, the overall proportion of death was $32.7 \%$ in 2006-2014. After controlling for potential confounders, age $\geqslant 75$ years, ESRD, malignancy, AFB-smear positivity, TB-culture positivity, pleural effusion on chest radiograph and notification by ordinary ward or intensive care units were associated with a higher risk of allcause death; while high education, a cavity on chest radiograph and directly observed therapy were associated with a lower risk of all-cause death.

This study found that the mortality was extremely high in elderly patients with TB, which was higher than $3.9 \%$ in Taiwanese elderly population without TB [12]. As compared with patients aged 65-74 years, patients aged 75 years and older had an even higher risk of TB-specific and non-TB-specific mortality. Higher mortality among older TB patients may be due to waning immunity and increased comorbidities.
Additionally, elderly patients with TB are more likely to experience a delay in TB diagnosis and treatment [13], which might cause the high mortality in this population. Since elderly individuals are at an increased risk of TB infection [1], clinicians need to be aware of TB in this population.

We identified individual socio-economic status variables that were risk factors for mortality. Specifically, individuals with at least a high school education were associated with a lower risk of death. Prior studies have reported that people with higher socio-economic status are more likely to receive superior treatment and additional diagnostic procedures [14]. Moreover, patients with low educational levels had a poor understanding of $\mathrm{TB}$, which resulted in a delay in diagnosis [15], interruption of treatment [16] and a higher rate of mortality [17]. To improve TB treatment outcomes in elderly individuals, future control programmes should particularly target patients with a lower educational level. 
This study found that a cavity on chest radiograph was associated with a lower risk of mortality. The protective effect of cavitary diseases on TB mortality in this study may reflect the fact that cavitary diseases raise the suspicion for TB in clinicians' minds and reduce diagnostic delay [18]. Since elderly patients with TB are more likely to have a non-specific clinical presentation at TB onset (e.g. no cavity lesions on chest radiograph) [4, 19], elderly patients suspected to have TB should be evaluated carefully to ensure early initiation of treatment.

This study showed that patients receiving directly observed therapy had a lower risk of mortality than those on self-administered therapy. In the Taipei DOTS programme, each directly observed treatment (DOT) observer monitors 5-10 patients with TB. DOT observers are trained to interview patients with TB about their TB symptoms and complications of treatment under the supervision of public health nurses. When patients with TB on DOT have, for example, worsened dyspnoea or blurred vision, public health nurses contact the doctors to arrange a hospital visit. Public health nurses daily check the regimen and dosage of TB drugs of each DOT patient according to TB treatment guidelines daily [20], and remind prescribing doctors, if needed, to ensure the patients are provided with appropriate TB drugs. DOT has been recommended for TB patients to improve treatment adherence [21]. Our study suggests that DOT programme should be applied to all elderly patients with TB to further reduce mortality.

Consistent with previous reports, long-term care facility residences [23], malignancy [24], ESRD [24], AFB-smear positivity [25], TB-culture positivity [24], pleural effusion on chest radiograph [24] and notification from an ordinary ward or intensive care unit [25] were associated with a higher risk of mortality.

The strengths of this study included that this was a city-wide population-based study with a large number of patients enrolled in the cohort, and treatment outcomes were tracked and recorded using standardised systems. However, several limitations should be considered when interpreting the findings of this study. First, this was a secondary-data study. Important information about TB patients, e.g. intravenous drug use, was not available in the surveillance data. Second, the cause of death among TB patients relied on ICD-9-CM codes in the Taiwan Death Certification Registry, and the outcome of cause of death may have been misclassified. This nondifferential misclassification of outcome would bias the results towards a null association. Finally, the external validity of our findings may be a concern because almost all our enrollees were Taiwanese. The generalisability of our results to other non-Asian ethnic groups requires further verification.

\section{CONCLUSIONS}

According to the above analysis, this study found that the proportion of death among elderly patients with TB in Taipei, Taiwan, was high. Age $\geqslant 75$ years, ESRD and malignancy were associated with a higher risk of all-cause mortality; while high education, a cavity on chest radiograph and directly observed therapy were associated with a lower risk of all-cause death. To improve TB treatment outcomes, future control programmes should particularly target individuals with comorbidities (e.g. ESRDs and malignancy) and those with a lower socio-economic status (e.g. not educated). Additionally, a directly observed therapy programme should be applied to all elderly patients with TB to further reduce mortality.

\section{ACKNOWLEDGEMENTS}

The authors are grateful to registered nurses Jen-Chieh Hsiao and Miao-Yun Chen for interviewing the subjects and reviewing their medical records.

\section{DECLARATION OF INTEREST}

None.

\section{REFERENCES}

1. World Health Organization (2014) Global tuberculosis control. WHO report 2014. (http://www.who.int/tb/publications/global_report/gtbr15_main_text.pdf). Accessed 20 September 2016.

2. Taiwan Centers for Disease Control and Prevention (2016) [Promulgated definitions of TB]. Taipei, Taiwan: CDC. (http://nidss.cdc.gov.tw/singledisease. aspx? $\mathrm{t}=\mathrm{s} \& \mathrm{dc}=1 \& \mathrm{dt}=3 \&$ disease $=010) \quad$ Accessed 16 September 2016. [Chinese].

3. Yen YF, et al. Risk factors for unfavorable outcome of pulmonary tuberculosis in adults in Taipei, Taiwan. Transactions of the Royal Society of Tropical Medicine and Hygiene 2012; 106: 303-308.

4. Cruz-Hervert LP, et al. Tuberculosis in ageing: high rates, complex diagnosis and poor clinical outcomes. Age and Ageing 2012; 41: 488-495.

5. Byng-Maddick R, et al. Does tuberculosis threaten our ageing populations? BMC Infectious Diseases 2016; 16: 119 . 
6. Morris CD. Pulmonary tuberculosis in the elderly: a different disease? Thorax 1990; 45: 912-913.

7. Schaaf HS, et al. Tuberculosis at extremes of age. Respirology 2010; 15: 747-763.

8. Balabanova Y, et al. The Directly Observed Therapy Short-Course (DOTS) strategy in Samara Oblast, Russian Federation. Respiratory Research 2006; 7: 44.

9. Department of Health. Vital Statistics in Taiwan. Taiwan: Department of Health, Executive Yuan, 1971.

10. Waitt CJ, et al. A systematic review of risk factors for death in adults during and after tuberculosis treatment. International Journal of Tuberculosis and Lung Disease 2011; 15: 871-885.

11. Jasmer RM, et al. Tuberculosis treatment outcomes: directly observed therapy compared with selfadministered therapy. American Journal of Respiratory and Critical Care Medicine 2004; 170: 561-566.

12. Ministry of Health and Welfare. Vital Statistics in Taiwan. Taiwan: Ministry of Health and Welfare, Executive Yuan. (http://www.mohw.gov.tw/CHT/DOS/ Statistic.aspx?f_list_no $=312 \&$ fod_list_no $=1601)$. Accessed 16 December 2016. [Chinese].

13. Storla DG, et al. A systematic review of delay in the diagnosis and treatment of tuberculosis. BMC Public Health 2008; 8: 15.

14. Younis MZ, et al. The impact of HMO and hospital competition on hospital costs. Journal of Health Care Finance 2005; 31: 60-74.

15. Hoa NP, et al. Knowledge about tuberculosis and its treatment among new pulmonary $\mathrm{TB}$ patients in the north and central regions of Vietnam. International Journal of Tuberculosis and Lung Disease 2004; 8: 603-608.
16. Elzinga G, et al. Scale up: meeting targets in global tuberculosis control. Lancet 2004; 363: 814-819.

17. Okanurak K, et al. Factors contributing to treatment success among tuberculosis patients: a prospective cohort study in Bangkok. International Journal of Tuberculosis and Lung Disease 2008; 12: 1160-1165.

18. Lin HP, et al. Diagnosis and treatment delay among pulmonary tuberculosis patients identified using the Taiwan reporting enquiry system, 2002-2006. BMC Public Health 2009; 9: 55.

19. Chiang CY, et al. Patient and health system delays in the diagnosis and treatment of tuberculosis in Southern Taiwan. International Journal of Tuberculosis and Lung Disease 2005; 9: 1006-1012.

20. Hopewell PC, et al. International standards for tuberculosis care. Lancet Infect Dis 2006; 6: 710-725.

21. Frieden TR, et al. Promoting adherence to treatment for tuberculosis: the importance of direct observation. Bulletin of the World Health Organization 2007; 85: 407-409.

22. Yen YF, et al. Association of body mass index with tuberculosis mortality: a population-based follow-up study. Medicine (Baltimore) 2016; 95: e2300.

23. Yen YF, et al. DOT associated with reduced all-cause mortality among tuberculosis patients in Taipei, Taiwan, 2006-2008. International Journal of Tuberculosis and Lung Disease 2012; 16: 178-184.

24. Kliiman K, et al. Predictors and mortality associated with treatment default in pulmonary tuberculosis. International Journal of Tuberculosis and Lung Disease 2010; 14: 454-463.

25. Pepper DJ, et al. Barriers to initiation of antiretrovirals during antituberculosis therapy in Africa. PLOS ONE 2011; 6: e19484. 